\title{
Determinants of Obesity Among Women of Childbearing Age in Urban Areas of Ethiopia
}

\author{
Wubegzier Mekonnen ${ }^{1,}$, Alemayehu Bogale ${ }^{2}$ \\ ${ }^{1}$ School of Public Health, College of Health Sciences, Addis Ababa University, Addis Ababa, Ethiopia \\ ${ }^{2}$ College of Health and Medical Sciences, Haramaya University, Harar, Ethiopia
}

Email address:

wubegizerm@gmail.com (W. Mekonnen), alemayehubog@gmail.com (A. Bogale)

${ }^{*}$ Corresponding author

\section{To cite this article:}

Wubegzier Mekonnen, Alemayehu Bogale. Determinants of Obesity Among Women of Childbearing Age in Urban Areas of Ethiopia. International Journal of Nutrition and Food Sciences. Vol. 6, No. 3, 2017, pp. 134-138. doi: 10.11648/j.ijnfs.20170603.14

Received: May 24, 2016; Accepted: June 1, 2016; Published: April 28, 2017

\begin{abstract}
Background: Obesity is a major public health problem in both developed and developing countries. Its prevalence is increasing rapidly and reached epidemic proportions globally. Evidence suggests that the situation is likely to get worse especially among women because women tend to gain greatest amount of weight during their child-bearing age, putting them at risk for cardiovascular disease, type II diabetes, hypertension, and many other chronic disorders. Only few studies assessed the situation of obesity in women of childbearing age living in urban areas of Ethiopia. Objectives: The aim of this study is to identify the determinant factors of obesity among women of childbearing age in urban areas of Ethiopia. Methods: The study used women's of childbearing age dataset from the 2011 Ethiopian Demographic and Health Survey. The survey sample was designed to provide national, urban/rural, and regional representative estimates of key health and demographic indicators. This study used 3,535 urban women's, from the total 14,505 eligible women of childbearing age in the country. The sample was selected using a two-stage stratified cluster sampling procedure. Odds ratio along with 95\% confidence interval in binary logistic regression was used to assess determinant factors associated with obesity among urban women of childbearing age. Results: The prevalence of obesity was (2.84\%) among women of childbearing age living in urban areas of Ethiopia. Obesity was significantly associated with occupation, age, marital status, children ever born, wealth terciles, religion, parent's educational status and residing region in the binary logistic regression analysis. Parents education $(\mathrm{AOR}=0.50$ : $95 \% \mathrm{CI}(0.26-$ 0.94), wealth tersiles ( $\mathrm{AOR}=2.01: 95 \% \mathrm{CI}(1.10-3.67)$ and $(\mathrm{AOR}=6.37: 95 \% \mathrm{CI}(3.38-12.00)$ in medium and high, respectively and residing regions $(\mathrm{AOR}=4.91$ : 95\% $\mathrm{CI} ; 1.24-19.47),(\mathrm{AOR}=5.68: 95 \% \mathrm{CI} ; 1.52-21.16),(\mathrm{AOR}=17.81: 95 \%$ $\mathrm{CI} ; 4.97$ - 63.78), (AOR=4.62: 95\% CI; 1.36 - 15.64), (AOR=4.21: 95\% CI; 1.28 - 13.75) and $(\mathrm{AOR}=6.06: 95 \% \mathrm{CI} ; 1.81-$ 20.23) in Afar, Oromiya, Somali, Harari, Addis Ababa and Dire Dawa regions, respectively were found to be determinants of obesity. Conclusion: The prevalence of obesity among women of childbearing age in urban areas was high. Parents' education, wealth terciles and residing region were associated with obesity. Targeted interventions should be put in place to reverse increasing levels of obesity in order to prevent the risk of obesity and its related life threatening effects.
\end{abstract}

Keywords: Obesity, Body Mass Index, Women of Childbearing Age, Urban, Ethiopia

\section{Introduction}

Obesity is the accumulation of total body fat. It is clinically defined as having a body mass index (BMI) of over $30 \mathrm{~kg} / \mathrm{m}^{2}$ [1]. More than 1.4 billion adults, 20 and older, were overweight. Of these overweight adults, over 200 million men and nearly 300 million women were obese [2, 3]. The prevalence of obesity is increasing rapidly in both developed and developing countries. It is reached epidemic proportions globally, and evidence suggests that the situation is likely to get worse especially among women. One of the reasons for this is because women tend to gain greatest amount of weight during their child-bearing age, putting them at risk for cardiovascular disease, type II diabetes, hypertension, and many other chronic disorders [4-8]. Obesity and its associated health effects are more prevalent in women than in men: with the female to male difference in obesity was $+4.6 \%$ [9]. 
More than 2.8 million people die each year as a result of being overweight or obese, and an estimated 35.8 million $(2.3 \%)$ of global DALYs are caused by overweight or obesity. Overweight and obesity lead to adverse metabolic effects on blood pressure, cholesterol, triglycerides and insulin resistance. Risks of coronary heart disease, ischemic stroke and type 2 diabetes mellitus increase steadily with increasing body mass index (BMI), a measure of weight relative to height. The worldwide prevalence of obesity has nearly doubled between 1980 and 2008. In 2008, 10\% of men and $14 \%$ of women in the world were obese (BMI $\geq 30$ $\mathrm{kg} / \mathrm{m} 2$ ), compared with $5 \%$ for men and $8 \%$ for women in 1980. An estimated 205 million men and 297 million women over the age of 20 were obese - a total of more than half a billion adults worldwide [10].

The data analysis on BMI was based on 14,505 women age 15-49 years. Of these BMI weighted women's, 3,535 were residing in urban areas. The mean BMI for Ethiopian women age $15-49$ is $20 \mathrm{~kg} / \mathrm{m}^{2}$. While Five percent were overweight, and just 1 percent were obese nationally. Obesity was varying with residence from $2.84 \%$ in urban areas and only $0.4 \%$ in rural areas. The prevalence of obesity was as high as $10.6 \%$ in Somali region and $0.7 \%$ in Tigray region [11]. Therefore, this study was conducted to identify the determinant factors of obesity among women of childbearing age in urban areas of Ethiopia.

\section{Methods}

This study used the 2011 Ethiopian National Demographic and Health Survey (EDHS) (11). The 2011 EDHS weighted the BMI of 14,505 women age 15-49 years nationally and 3,535 from urban residence of the country. Two-stage stratified sampling procedure was used to select 624 clusters in the first stage from the list of enumeration areas delineated during the 2007 population and housing census (12). EDHS aimed at providing national, urban/rural, estimates of health and demographic parameters. The ICF International DHS questionnaire was adopted by Central Statistical Agency. Data were downloaded from measure DHS website with the consent of ICF international.

The dependent variable for this study was obesity while the main independent variables are region, BMI of mothers, mother's education and household wealth quintile. Data were analyzed using STATA version 11 . Obesity prevalence rate was computed. Odds ratio along with $95 \%$ confidence interval in binary logistic regression was used to assess the association between independent variables and obesity prevalence. Ethical clearance was sought from appropriate bodies.

\section{Results}

A total of 3, 535 women of childbearing age living in urban areas of Ethiopia were included in the survey. The majority of the study participants $1,993(56.4 \%)$ were not in union in their marital status. Nearly two third of women 2,322 (65.7\%) were Orthodox while 595 (16.8\%), and 618
(17.5\%) were Muslim and other Christians, respectively. More than one fifth $758(21.5 \%)$ of the study subjects could not read and write during the time of the survey. More than one third 1,492 (42.2\%) of the study subjects were no occupation. Nearly one third of the respondents 1,185 $(33.5 \%)$ belonged to households in the lowest wealth terciles. With regard to age of the study subjects more than one third $1,393(39.4 \%)$ were in the age range of 20 to 29 . The majority $3,156(89.3 \%)$ of the study subjects had less than five children ever born (Table 1).

Table 1. Socio-demographic characteristics of study participants among urban areas of Ethiopia, 2011.

\begin{tabular}{llll}
\hline Variables & & Frequency $(\mathbf{n = 3 , 5 3 5})$ & Percent \\
\hline \multirow{2}{*}{ Marital status } & Married & 1,542 & 43.6 \\
& Not in union & 1,993 & 56.4 \\
Religion & Orthodox & 2,322 & 65.7 \\
& Muslim & 595 & 16.8 \\
& Other Christians & 618 & 17.5 \\
Educational & No education & 758 & 21.5 \\
status & Primary & 1,521 & 43 \\
& Secondary & 719 & 20.3 \\
& Higher & 537 & 15.2 \\
& No occupation & 1,492 & 42.2 \\
Occupation & Employee & 343 & 9.7 \\
& Merchant & 1,076 & 30.4 \\
& Farmer & 212 & 6 \\
& Daily laborer & 412 & 11.7 \\
Wealth terciles & Low & 1,185 & 33.5 \\
& Medium & 1,172 & 33.2 \\
& High & 1,178 & 33.3 \\
Age & $15-19$ & 993 & 28.1 \\
& $20-29$ & 1,393 & 39.4 \\
bhildren ever & < & 764 & 21.6 \\
& > 5 & 385 & 10.9 \\
\hline
\end{tabular}

From the total 3,535 study subjects, 100 (2.84\%) of women of childbearing age living in urban areas of Ethiopia were obese during the interview. The prevalence of obesity significantly varied with occupation. Obesity was $27(7.7 \%)$, $36(2.4 \%), 28(2.6 \%)$, and $10(2.5 \%)$ in employee, no occupation, merchant, and daily laborer, respectively. The obesity prevalence rate was significantly higher among women's whose age was older than 40 years as compared to younger once less than 20 years $(8.9 \%$ and $0.3 \%)$. Obesity was significantly higher among married women as compared to those not in unions ( $5 \%$ and $1.2 \%$ ). Meanwhile, obesity was as high as $5.8 \%$ in women's who ever born greater or equal to five children as compared to women's who ever born less than five children $(2.5 \%)$. Obesity was $5.4 \%, 2 \%$ and $1.2 \%$ among women whose wealth terciles was high, medium and low, respectively. Obesity was 5.1\% among Muslim women as compared to $2.6 \%$ in women's confessed in Orthodox religion. In the same fashion, obesity was $7.8 \%$ in women's having secondary education where as $2 \%$ in women's who were no formal education. On the other hand, obesity significantly varied with residing region. It was as high as $10.6 \%$ in women's residing in Somali region while it was very low $0.7 \%$ in Tigray region (Table 2 ). 
Table 2. Patterns of Obesity by Basic Background Characteristics of Women's of child bearing age in urban areas of Ethiopia, 2011.

\begin{tabular}{|c|c|c|c|}
\hline \multirow{2}{*}{ Characteristics } & \multicolumn{2}{|l|}{ Obesity } & \multirow{2}{*}{$\begin{array}{l}\text { P- } \\
\text { value }\end{array}$} \\
\hline & Yes (\%) & Number $(n=3,535)$ & \\
\hline \multicolumn{4}{|l|}{ Occupation } \\
\hline No occupation & 2.4 & 1,492 & \multirow{5}{*}{0.00} \\
\hline Employee & 7.7 & 343 & \\
\hline Merchant & 2.6 & 1,076 & \\
\hline Farmer & 0 & 212 & \\
\hline Daily laborer & 2.5 & 412 & \\
\hline \multicolumn{4}{|l|}{ Age } \\
\hline $15-19$ & 0.3 & 993 & \multirow{4}{*}{0.00} \\
\hline $20-29$ & 1.6 & 1,393 & \\
\hline $30-39$ & 5.3 & 764 & \\
\hline $40-49$ & 8.9 & 385 & \\
\hline \multicolumn{4}{|l|}{ Marital status } \\
\hline Married & 5 & 1,542 & \multirow[t]{2}{*}{0.00} \\
\hline Not in Union & 1.2 & 1,993 & \\
\hline \multicolumn{4}{|l|}{ Children ever born } \\
\hline$<5$ & 2.5 & 3,156 & \multirow[t]{2}{*}{0.00} \\
\hline$>=5$ & 5.8 & 379 & \\
\hline \multicolumn{4}{|l|}{ Wealth terciles } \\
\hline Low & 1.2 & 1,185 & \multirow{3}{*}{0.00} \\
\hline Medium & 2 & 1,172 & \\
\hline High & 5.6 & 1,178 & \\
\hline \multicolumn{4}{|l|}{ Religion } \\
\hline Orthodox & 2.6 & 2,322 & \multirow{3}{*}{0.01} \\
\hline Muslim & 5.1 & 595 & \\
\hline Other Christians & 1.6 & 618 & \\
\hline \multicolumn{4}{|c|}{ Parent's educational status } \\
\hline No education & 2 & 437 & \multirow{4}{*}{0.00} \\
\hline Primary & 3.6 & 809 & \\
\hline Secondary & 7.8 & 380 & \\
\hline Higher & 5.7 & 384 & \\
\hline \multicolumn{4}{|l|}{ Region } \\
\hline Tigray & 0.7 & 275 & \multirow{11}{*}{0.00} \\
\hline Affar & 4.2 & 35 & \\
\hline Amhara & 1.2 & 870 & \\
\hline Oromiya & 3.5 & 841 & \\
\hline Somali & 10.6 & 114 & \\
\hline Benishangul Gumuz & 2.6 & 35 & \\
\hline SNNP & 1.5 & 472 & \\
\hline Gambella & 2.2 & 19 & \\
\hline Harari & 4.9 & 26 & \\
\hline Addis Ababa & 4.1 & 805 & \\
\hline Dire Dawa & 6.2 & 43 & \\
\hline
\end{tabular}

Determinants of obesity

Parents' education, wealth terciles and residing region were found to be the determinant factors of obesity among women of childbearing age in urban areas of Ethiopia. Women whose parents have no formal education were $50 \%$ times less likely $\mathrm{AOR}=0.50: 95 \% \mathrm{CI}(0.26-0.94)$ to be obese compared to those having higher education. Obesity was significantly associated with wealth terciles, women's whose wealth terciles were in medium were more than two times more likely ( $\mathrm{AOR}=2.01: 95 \% \mathrm{CI}(1.10-3.67)$ to be obese as compared to the low wealth terciles. Meanwhile, women's whose wealth terciles were high were more than six times $(\mathrm{AOR}=6.37: 95 \% \mathrm{CI}(3.38-12.00)$ more likely to be obese as compared to the low wealth terciles. On the same way, obesity was significantly associated with residing region. Women's residing in $\mathrm{Afar}(\mathrm{AOR}=4.91: 95 \% \mathrm{CI} ; 1.24$
- 19.47), Oromiya (AOR=5.68: 95\% CI; 1.52 -21.16), Somali (AOR $=17.81$ : 95\% CI; 4.97 - 63.78), Harari $(\mathrm{AOR}=4.62: \quad 95 \% \quad \mathrm{CI} ; 1.36$ - 15.64), Addis Ababa $(\mathrm{AOR}=4.21: 95 \% \quad \mathrm{CI} ; \quad 1.28-13.75)$ and Dire Dawa (AOR=6.06: 95\% CI; $1.81-20.23$ ) were found to be obese as compared to women's residing in Tigray (Table 3 ).

Table 3. The Association between background characteristics and obesity among women of child bearing age living in urban areas of Ethiopia, 2011.

\begin{tabular}{|c|c|c|c|c|}
\hline \multirow{2}{*}{ Variables } & \multicolumn{2}{|c|}{ Obesity } & \multicolumn{2}{|l|}{ OR $(95 \%) C I$} \\
\hline & Yes & No & Crude & Adjusted \\
\hline \multicolumn{5}{|l|}{$\begin{array}{l}\text { Family } \\
\text { planning use }\end{array}$} \\
\hline No & 63 & 2,451 & 1 & 1 \\
\hline Yes & 37 & & $1.72(1.29,2.31)^{*}$ & $0.93(0.67,1.30)$ \\
\hline $\begin{array}{l}\text { Parent } \\
\text { education }\end{array}$ & & 984 & & \\
\hline No education & 8 & & $0.42(0.25,0.69) *$ & $0.50(0.26,0.94)^{* *}$ \\
\hline Primary & 29 & 428 & $0.53(0.35,0.80)^{*}$ & $0.76(0.46,1.26)$ \\
\hline Secondary & 30 & 780 & $0.74(0.49,1.11)$ & $0.72(0.46,1.14)$ \\
\hline Higher & 22 & 350 & 1 & 1 \\
\hline Religion & & 362 & & \\
\hline Orthodox & 60 & & 1 & 1 \\
\hline $\begin{array}{l}\text { Other } \\
\text { Christians }\end{array}$ & 10 & 2,261 & $0.94(0.58,1.51)$ & $0.86(0.48,1.57)$ \\
\hline Muslim & 30 & 608 & $1.53(1.13,2.08) *$ & $1.47(0.99,2.18)$ \\
\hline $\begin{array}{l}\text { Frequency of } \\
\text { watching } \\
\text { television }\end{array}$ & & 565 & & \\
\hline Not at all & 32 & & 1 & 1 \\
\hline $\begin{array}{l}\text { Less than once } \\
\text { a week }\end{array}$ & 30 & 809 & $0.75(0.52,1.07)$ & $0.71(0.47,1.08)$ \\
\hline $\begin{array}{l}\text { At least once a } \\
\text { week }\end{array}$ & 39 & 1,308 & $0.77(0.54,1.08)$ & $0.71(0.47,1.07)$ \\
\hline $\begin{array}{l}\text { Educational } \\
\text { status of } \\
\text { women }\end{array}$ & & 1,318 & & \\
\hline No education & 26 & & $1.05(0.69,1.61)$ & $1.25(0.65,2.38)$ \\
\hline Primary & 28 & 733 & $0.55(0.36,0.84)^{*}$ & $0.87(0.50,1.52)$ \\
\hline Secondary & 27 & 1,492 & $1.00(0.65,1.53)$ & $1.33(0.79,2.24)$ \\
\hline Higher & 19 & 692 & 1 & 1 \\
\hline Wealth terciles & & 517 & & \\
\hline Low & 14 & & 1 & 1 \\
\hline Medium & 24 & 1,171 & $1.46(0.86,2.49)$ & $2.01(1.10,3.67)^{* *}$ \\
\hline High & 63 & 1,149 & $3.05(1.90,4.92)^{*}$ & $6.37(3.38,12.00)^{* *}$ \\
\hline Region & & 1,115 & & \\
\hline Tigray & 2 & & 1 & 1 \\
\hline Afar & 1 & 273 & $5.38(1.46,19.79)^{*}$ & $4.91(1.24,19.47)^{* *}$ \\
\hline Amhara & 11 & 34 & $2.00(0.44,9.05)^{*}$ & $1.52(0.24,9.44)$ \\
\hline Oromiya & 29 & 859 & $4.20(1.16,15.19)^{*}$ & $5.68(1.52,21.16)^{* *}$ \\
\hline Somali & 12 & 811 & $12.87(3.83,43.25)^{*}$ & $\begin{array}{l}\text { 17.81(4.97 } \\
63.78)^{* *}\end{array}$ \\
\hline $\begin{array}{l}\text { Benishangul } \\
\text { Gumuz }\end{array}$ & 1 & 102 & $3.16(0.70,14.32)$ & $4.41(0.93,20.78)$ \\
\hline SNNPR & 7 & 34 & $2.26(0.50,10.23)$ & $2.86(0.60,13.60)$ \\
\hline Gambella & 0 & 465 & $3.01(0.71,12.73)^{*}$ & $1.95(0.37,10.11)$ \\
\hline Harari & 1 & 18 & $5.92(1.78,19.66)^{*}$ & $4.62(1.36,15.64)^{* *}$ \\
\hline Addis Ababa & 33 & 25 & $5.33(1.66,17.04)^{*}$ & $4.21(1.28,13.75)^{* *}$ \\
\hline Dire Dawa & 3 & $\begin{array}{l}773 \\
41\end{array}$ & $8.38(2.57,27.27)^{*}$ & $6.06(1.81,20.23)^{* *}$ \\
\hline
\end{tabular}

${ }^{* *}$ Statistically significant association at $5 \%$ level of significance.

\section{Discussion}

This study was conducted with the aim of identifying the 
determinant factors of obesity among women of childbearing age in urban areas of Ethiopia. The study revealed that about $2.8 \%$ of the respondents were found to be obese. The obesity prevalence rate estimated in this study was nearly threefold times higher than the national estimate according to the 2011 Ethiopia Demographic and Health Survey report (11) and also higher than the study conducted among Nepal adult women and young adults in Uganda $(13,14)$. But lower than the studies conducted in Central Pennsylvania, Cameroon and Ghana (15-17). The variation in the level of obesity might be attributed to cultural, residence, food preferences, working habits and living conditions of the respondents.

In the binary logistic regression analysis, obesity was significantly associated with occupation, age, marital status, children ever born, wealth terciles, religion, parent's educational status and residing region.

In this study parents' education, wealth terciles, and residing region were found to be the determinant factors of obesity among women of childbearing age in urban areas of Ethiopia.

Moreover, obesity was significant in women's whose wealth tereciles were medium and high. This finding is also consistent with the study conducted in Uganda, Ghana and global studies (14, 18 and 19).

This study revealed that obesity was significantly associated with residing region among women of childbearing age in urban areas of Ethiopia. The finding is consistent with the study conducted in Uganda where obesity was varying in residing cities of the country (14). This could be partly explained by variations in climatic conditions, food preferences, livelihood activities and genetic factors.

\section{Conclusion}

The study revealed that the prevalence of obesity in urban areas of Ethiopia is high as compared to other rural areas of the country. Parents' education, wealth terciles and residing region were found to be significant predictors of obesity among women of child bearing age living in urban areas of Ethiopia. Targeted interventions should be put in place to reverse increasing levels of obesity in order to prevent the risk of obesity and its related life threatening health effects.

\section{Acknowledgement}

We are grateful to the women and people who participated in this study.

\section{Competing Interest}

We declare that we have no conflict of interest.

\section{Authors' Contributions}

$\mathrm{WM}$ and $\mathrm{AB}$ carried out the study, participated in the analysis and manuscript drafting. Both authors read and approved the final manuscript.

\section{References}

[1] Houston D K, Nicklas BJ, Zizza CA. Weighty concerns: The growing Assoc prevalence of obesity among older adults. J A m Diet Assoc 2009; 109:1886- 9.

[2] Berghöfer A, Pischon T, Reinhold T, Apovian CM, Sharma AM, Willich SN. Obesity prevalence from a European perspective: a systematic review. BMC Public Health 2008 5;8:200. doi: 10.1186/1471-2458-8-200.

[3] Sidik SM, Rampal L. The prevalence and factors associated with obesity among adult women in Selangor, Malaysia. Asia Pacific Family Medicine 2009, 8:2 doi:10.1186/1447-056X-8-2.

[4] Price RA, Danielle RR, Nicholas JG. Resemblance for Body Mass Index in Families of Obese African American and European American Women. Obesity Research. 2000; 8: 360-366.

[5] Siega-Riz AM, Evenson KR, Dole N: Pregnancy-related Weight Gain- A Link to Obesity? Nutrition Reviews 2004, 62(7): S105-S111.

[6] World Health Organization. Obesity and overweight 2012.

[7] Puhl RM, Heuer CA. Obesity stigma: Important considerations for public health. American Journal of Public Health 2010; 100: 1019-1028.

[8] Patterson RE, Frank LL, Kristal AR, White E. A comprehensive examination of health conditions associated with obesity in older adults. Am J Prev Med 2004; 27: 385-390.

[9] Mascie-Taylor CG, Goto R. Human variation and body mass index: A review of the universality of BMI cut-offs, gender, and urban-rural differences, and secular changes. J Physiol Anthropol 2007; 26: 109-112.

[10] World Health Organization, Global Health Observatory (GHO), Obesity: Situation and Trend 2012. http://www.who.int/gho-/ncd/risk_factors/obesity text/en/index.html.

[11] Central Statistical Agency [Ethiopia] and ICF International: Ethiopia Demographic and Health Survey 2011. Addis Ababa, Ethiopia and Calverton, Maryland, USA: Central Statistical Agency and ICF International; 2012.

[12] Central Statistics Agency, Population Census Commission: Summary and Statistical Report of the 2007. Addis Ababa: Population and Housing Census; 2008.

[13] Shahi M, Rai L, Adhikari DR, Sharma M. Prevalence and factors associated with obesity among adult women of Nepal. Global Journal of Medicine and Public Health 2013, 2:4.

[14] Baalwa J, Byarugaba BB, Kabagambe KE, Otim AM. Prevalence of overweight and obesity in young adults in Uganda. African Health Sciences 2010; 10(4): 367-373.

[15] Geyer N. Overweight and Obesity in the Reproductive-Age Population of the Central Pennsylvania Women's Health Study. J Obes Wt Loss Ther 2013, 3: 158. doi: 10.4172/21657904.1000158.

[16] Kamadjeu MR, Edwards R, Atanga SJ, Kiawi CE, Unwin N, Mbanya J. Anthropometry measures and prevalence of obesity in the urban adult population of Cameroon: an update from the Cameroon Burden of Diabetes Baseline Survey. BMC Public Health 2006, 6:228 doi: 10.1186/1471-2458-6-228. 
[17] Agbeko PM, Akwasi K, Andrews AD, Gifty BO. Predictors of Overweight and Obesity among Women in Ghana. The Open Obesity Journal, 2013, 5, 72-81.

[18] Pobee RA, Owusu WB, and Plahar WA. The prevalence of obesity among female teachers of child-bearing age in Ghana. African Journal of Food, Agriculture, Nutrition and Development; 13 (2): 2013.
[19] Moore S, Hall NJ, Harper S, Lynch WJ. Global and National Socioeconomic Disparities in Obesity, Overweight, and Underweight Status. Journal of Obesity 2010; doi: $10.1155 / 2010 / 514674$. 